\title{
Corrección de una clase III esqueletal con anclaje óseo en paciente en crecimiento: Reporte de caso
}

\section{Skeletal class III correction in growing patient with bone anchorage: Case Report}

\author{
Paloma González Higuera ${ }^{1}$ (D) | Laura Mónica López Pérez Franco ${ }^{2}$ (D) | Francisco Alejandro Díaz Galindo ${ }^{3}$ \\ | Yareli Hernández Ávila ${ }^{1}$ (D) | Marco Felipe Salas Orozco ${ }^{2}$ (D) | Miguel Angel Casillas Santana ${ }^{1}$ (D)
}

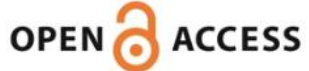

Afiliación Institucional

${ }^{1}$ Benemérita Universidad Autónoma de Puebla. Facultad de Estomatología, Departamento de Ortodoncia, Puebla, México.

${ }^{2}$ Universidad Autónoma de San Luis de Potosí, Facultad de Estomatología, Departamento de Ortodoncia y Ortopedia Dentomaxilofacial, San Luis Potosí, México.

Citaçión:

González-Higuera P, López-Pérez-Franco LM, Díaz-Galindo FA, Hernández-Ávila Y, Salas Orozco MF, Casillas-Santana MA. Corrección de una clase III esqueletal con anclaje óseo en paciente en crecimiento: Reporte de Caso. Rev Estomatol. 2021 Setembro 2021;29(2):e11286. DOI: 10.25100/re.v29i1.11286

Recibido: 25 Mayo de 2021

Evaluado: 3 Junio de 2021

Aceptado: 12 Julio de 2021

Publicado: 03 Noviembre 2021

Correspondence:

Miguel Ángel Casillas Santana. Calle 31 Poniente No. 1304 Colonia Los Volcanes CP. 72410, Puebla, México. Contacto: +52 44484676 45. Email: miguel.casillas@correo.buap.mx

Copyright:

(c) Universidad del Valle.

\section{RESUMEN}

Antecedentes: La clase III esqueletal, es una deformidad dentofacial donde el tercio inferior de la cara es más prominente, el tratamiento se decide según la etiología y la edad del paciente; si se encuentra en crecimiento la malformación puede ser tratada con un protocolo interceptivo y si es posible evitar la cirugía ortognática a futuro.

Objetivo: Mejorar la clase esqueletal, descruzar la mordida u obtener mordida borde a borde, mejorar la posición del labio superior y evaluar el comparativo inicial-final de SNA y ANB.

Reporte de caso: Paciente masculino de 13 años, sin antecedentes personales patológicos o familiares reportados; presenta clase III esqueletal responsiva bimaxilar, crecimiento vertical, clase molar I y canina III; fue tratado con el protocolo de mini placas BAMP (bone anchored maxillary protraction) por sus siglas en inglés, elásticos intermaxilares y un paladar con pistas planas.

Resultados: La fase ortopédica duro cinco meses y se logró mordida borde a borde y clase I esqueletal.

Discusión: Se obtuvieron resultados con el uso de mini implantes sin anclaje extraoral en menos tiempo a comparación de otros métodos que tienen que ser usados por 9-12 meses.

Conclusión: El protocolo BAMP puede ser usado en pacientes en crecimiento sin máscara facial para corregir la clase III esqueletal..

\section{PALABRAS CLAVE}

Clase III esqueletal; ortopedia dental; protracción maxilar; anclaje óseo; tratamiento temprano.

\section{ABSTRACT}

Background: Skeletal class III is a dentofacial deformity where the lower third of the face is more prominent. The treatment is decided according to the etiology and age of the patient; If the patient is in growing, the malformation can be treated with an interceptive protocol and if possible, avoid a orthognathic surgery in the future.

Objective: Improve the skeletal class, uncross the bite or obtain an edge-to-edge bite, improve the position of the upper lip and compare the initial-final relationship of ANS and ANB.

Case report: 13-year-old male patient, with no reported pathological or family history; presents skeletal class III, vertical growth, molar class I and canine III; he was treated with the protocol of mini BAMP (bone anchored maxillary protraction) plates, intermaxillary elastics and a palate with flat tracks.

Results: The orthopedic phase lasted five months and an edge-to-edge bite and skeletal class I were achieved. Discussion: Results were obtained with the use of mini plates without extraoral anchorage in less time compared to other methods that have to be used for 9-12 months.

Conclusion: The BAMP protocol can be used in growing patients without a face mask to correct skeletal class III.

\section{KEY WORDS}

Skeletal class III; dental orthopedics; maxillary protraction; bone anchorage; early treatment. 


\section{RELEVANCIA CLÍNICA}

Conocer las alternativas de tratamiento interceptivo que existen hoy en día para los pacientes clase III en crecimiento; así como el momento más oportuno para intervenir y prevenir los efectos adversos dentales y esqueletales.

\section{INTRODUCCIÓN}

La clase III esqueletal ha sido descrita como una deformidad dentofacial relacionada a una maloclusión clase III y mordida cruzada anterior; se considera que su etiología es hereditaria o relacionada a problemas endócrinos o factores ambientales; tiene una incidencia del $4 \%$ en caucásicos, $14 \%$ en asiáticos, $4.2 \%$ en estadounidenses y $17 \%$ en población mexicana, con mayor prevalencia en hombres y población hispana. ${ }^{1-4}$ Puede ser originada por responsiva de retrusión maxilar, anteroposición mandibular o ambas, para esto es importante realizar la cefalometría y determinar si es una clase III falsa o verdadera. ${ }^{2,5}$

El diagnóstico temprano de las discrepancias esqueletales permite un tratamiento interceptivo con la finalidad mejorar la condición actual, evitar o disminuir la complejidad de una cirugía ortognática a futuro o en el mejor de los casos, corregir la discrepancia en su totalidad. ${ }^{6,7}$ Este tipo de tratamiento esta indicado para pacientes que se encuentran en crecimiento; sin embargo, es necesario realizar estudios para valorar la edad cronológica y dental del paciente; la cual generalmente coincide en niños con crecimiento normal, no obstante, en algunos casos puede diferir; es por esto que existen parámetros para verificar si el paciente es candidato para este tipo de intervención: Etapas de calcificación radicular, análisis óseo de falanges y cervicales, desarrollo de caracteres sexuales secundarios, altura y peso. ${ }^{6-9}$

Una vez establecido el diagnóstico; se debe elegir algunas de las alternativas a seguir para este tipo de discrepancias; plano inclinado anterior, Frankel III, pistas planas clase III y siendo el más común la máscara facial; la cual aplica una fuerza sagital y genera una protracción del maxilar, sin embargo, se han reportado diversos efectos dentales indeseables. ${ }^{1,2,10}$
Recientemente, se ha descubierto que con la edad disminuyen los cambios dentales y aumentan los cambios esqueletales, es por esto que De Clerck et al., ${ }^{11,12}$ presentaron una técnica de anclaje óseo con mini placas en los maxilares (en superior, en la cresta infracigomática del arbotante maxilar y en inferior entre el lateral y el canino inferior permanente) conectados mediante elásticos vector clase III; y obtuvieron resultados favorables para resolver o disminuir la discrepancia esqueletal en pacientes entre los 10 y 12 años.(11) No obstante, las mini placas destinadas para este tratamiento son costosas por lo que se han realizado protocolos con placas rectas modificadas y de igual forma han reportado buenos resultados. ${ }^{11}$

\section{REPORTE DEL CASO}

\section{Diagnóstico}

Paciente masculino de 13 años referido a la Clínica de Ortodoncia de la Benemérita Universidad Autónoma de Puebla por presentar mordida cruzada anterior, clase molar I y canina III de Angle. Al realizar la historia clínica reporta un estado de salud aparentemente sano, sin datos patológicos familiares ni personales, manifestaciones de dolor en la articulación temporomandibular y tratamientos dentales previos negados.

El análisis de las fotografías faciales reveló un paciente dolicofacial, con forma de cara ovalada, perfil recto con labios competentes y labio superior ligeramente retruído, línea media facial coincidente con la línea media dental superior y leve laterognasia hacia la izquierda.

A la exploración intraoral se observa apiñamiento nulo superior e inferior, mordida cruzada anterior, línea media inferior desviada a la izquierda, clase molar I, canina III bilateral y ausencia de los segundos premolares superiores (Figura 1).

En la ortopantomografía se observó asimetría de las ramas, segundos premolares superiores retenidos, relación corona-raíz 1:2, múltiples restauraciones dentales y presencia de los cuatro terceros molares (Figura 1).

En el análisis cefalométrico, se obtuvo una clase III esqueletal por responsiva bimaxilar, patrón de crecimiento vertical, hiperplasia mandibular, proinclinación del incisivo superior y retroinclinación del incisivo inferior. 
Como parte del diagnóstico y parte importante para determinar el tipo de tratamiento, con la finalidad de evitar más radiación al paciente; en la radiografía lateral de cráneo se realizó el método para valorar el estado de maduración ósea vertebral propuesto por Baccetti y Franchi para determinar la relación del crecimiento óseo y edad cronológica; el paciente fue clasificado en un estadio $\mathrm{CS} 3$ debido a las concavidades presentes en $\mathrm{C} 2-\mathrm{C} 3$, forma trapezoidal en $\mathrm{C} 3-\mathrm{C} 4$; lo cual indica que el pico del crecimiento mandibular sucederá un año después a esta etapa (Tabla 1). ${ }^{13}$

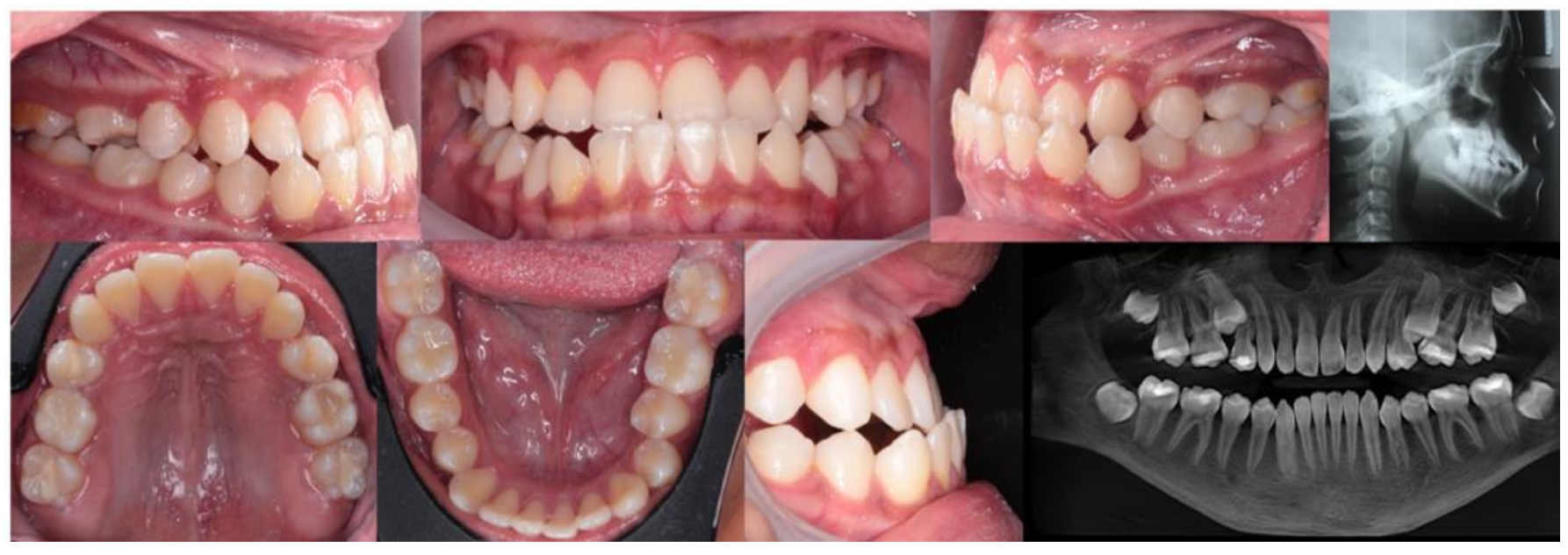

Figura 1. Fotos intraorales y radiografías iniciales.

Tabla 1. Datos cefalométricos iniciales y finales

\begin{tabular}{|c|c|c|c|}
\hline & Norma & Inicial & Final \\
\hline SNA & $82^{\circ}$ & $79^{\circ}$ & $83^{\circ}$ \\
\hline SNB & $80^{\circ}$ & $81^{\circ}$ & $80^{\circ}$ \\
\hline ANB & $2^{o}$ & $-2^{o}$ & $3^{\circ}$ \\
\hline \multicolumn{4}{|l|}{ Maxilar y mandíbula } \\
\hline SN-Plano Oclusal & $14^{\circ} \pm 2$ & $19^{\circ}$ & $20^{\circ}$ \\
\hline SN- GoGn & $32^{\circ} \pm 2$ & $37^{\circ}$ & $40^{\circ}$ \\
\hline \multicolumn{4}{|l|}{ Patrón de crecimiento } \\
\hline Eje facial (Ricketts) & $90^{\circ} \pm 3$ & $93^{\circ}$ & $92^{\circ}$ \\
\hline Altura facial inferior (Ricketts) & $47^{\circ} \pm 4$ & $43^{\circ}$ & $43.5^{\circ}$ \\
\hline \multicolumn{4}{|l|}{ Dental } \\
\hline ENA-ENP / Incisivo superior & $70^{\circ} \pm 2$ & $64^{\circ}$ & $60^{\circ}$ \\
\hline Incisivo inferior / Plano mandibular & $90^{\circ} \pm 2$ & $86^{\circ}$ & $87^{\circ}$ \\
\hline \multicolumn{4}{|l|}{ Facial } \\
\hline Labio superior (Ricketts) & $-5 \mathrm{~mm}$ & $-5 \mathrm{~mm}$ & $-1.5 \mathrm{~mm}$ \\
\hline Labio inferior (Ricketts) & $1 \mathrm{~mm}$ & $1 \mathrm{~mm}$ & $.5 \mathrm{~mm}$ \\
\hline
\end{tabular}




\section{Objetivos del tratamiento}

De acuerdo con el diagnóstico, se decidió mejorar la clase esqueletal, descruzar la mordida u obtener mordida borde a borde, mejorar la posición del labio superior y evaluar la relación inicial-final de SNA y ANB.

\section{Plan de tratamiento}

Se sugirieron dos alternativas de tratamiento basados en el diagnóstico y edad del paciente; la primera, esperar a completar el crecimiento y realizar un tratamiento ortodóncico prequirúrgico para cirugía ortognática y la segunda, un tratamiento interceptivo de pronóstico reservado con mini placas modificadas basado en el protocolo de protracción maxilar con anclaje óseo propuesto por De Clerck; con la posibilidad de mejorar la discrepancia dental y esqueletal, sin embargo, la laterognasia solo podría corregirse por procedimiento quirúrgico, los padres del paciente eligieron la segunda opción.

\section{Progreso del tratamiento}

Para colocar las mini placas, se realizó el procedimiento quirúrgico bajo anestesia local de articaina al $4 \%$ con abordaje lineal en fondo de saco a la altura del primer y segundo molar superior derecho e izquierdo, se realizó colgajo mucoperióstico, se expuso el arbotante cigomaticomaxilar donde se colocaron 4 placas rectas modificadas de 4 orificios, las cuales se fijaron con 2 tornillos monocorticales de $5 \mathrm{~mm}$ (Medartis sistema Modus ${ }^{\circledR}$ Trauma 2.0 de titanio) (titanio puro con clave ASTM F67, ISO 5832-2); es importante mencionar que todos los materiales de titanio utilizados en este procedimiento son biocompatibles, resistentes a la corrosión y no tóxicos en un ambiente bioógico; después se realizó abordaje lineal en fondo de saco de la zona mandibular a la altura del primer premolar y canino, se realizó colgajo mucoperióstico donde se colocaron dos placas rectas de 4 orificios y se fijaron con dos tornillos monocorticales autorroscantes de $5 \mathrm{~mm}$ por lado.

Se procedió al cierre de heridas con sutura de ácido poliglicólico de 4 0's, hemostasia, lavado profuso y se recetó analgésico y colutorios de Clorhexidina al $0.12 \%$ cada 12 horas después de la higiene oral por una semana.
Siete días después, se realizó el retiro de puntos de sutura y se valoró estabilidad de las miniplacas. Una semana después se empezó la fase ortopédica, en la misma consulta; se cementaron las pistas planas de acrílico y se inició con elásticos intermaxilares de 1/4 2oz (Ormco,USA) por dos semanas de los mini implantes superiores a inferiores (Figura 2).

En la consulta de seguimiento, se observó mejoría en sentido sagital pero la desviación hacia la izquierda de la línea media dental se mantuvo, esto debido a la laterognasia, sin embargo, se colocaron elásticos con fuerza asimétrica: $1 / 46 \mathrm{oz}$ (Ormco, USA) del lado derecho y $1 / 44.5 \mathrm{oz}$ (Ormco, USA) del lado izquierdo.

Se indicaron todas las consultas de seguimiento cada dos semanas y se mantuvieron los elásticos con fuerzas asimétricas hasta finalizar el tratamiento, cada consulta se checaban interferencias oclusales de las pistas planas y se desgastaban para permitir la mecánica de deslizamiento; se continuó con la misma mecánica durante el tiempo total de la fase ortopédica.

\section{Resultados}

Los resultados se obtuvieron con el análisis y comparación de la fotografías y cefalometría inicial y final (Figura 3). El tiempo total de la fase ortopédica fue de cinco meses y se logró relación borde a borde incisal y mejoría en la clase canina (Figura 3). En la cefalometría se obtuvo una anteroposición maxilar y una clase esqueletal I (ANB $3^{\circ}$ ) por una mejoría en el ángulo SNA $\left(83^{\circ}\right)$ y en el plano estético de Ricketts (-1.5 mm) (Tabla 1) (Figura 4).

Para finalizar la fase ortopédica, se retiraron las pistas planas y se remitió al departamento de cirugía maxilofacial para evaluar la estabilidad de las mini placas y viabilidad para seguir utilizándolas durante la segunda fase de tratamiento que corresponde a la parte ortodóncica, para la cual serán indicadas las extracciones de los segundos premolares superiores y primeros premolares inferiores.

\section{DISCUSIÓN}

El diagnóstico temprano de una clase III esqueletal permite 
B)

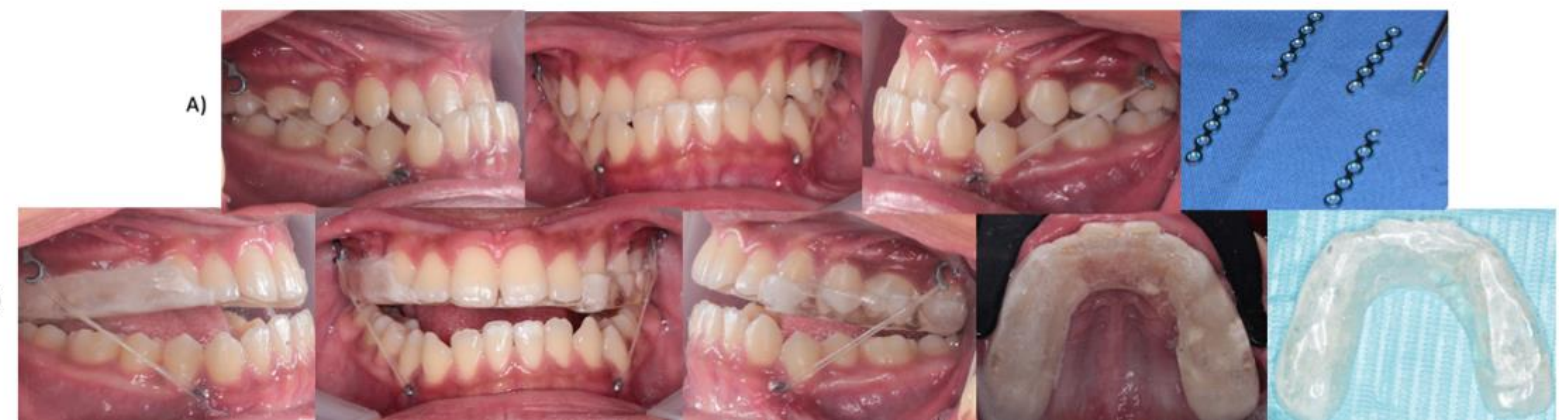

Figura 2. A) Colocación de mini placas modificadas y mini placas rectas modificadas. B) Activación de mini placas y paladar con pistas planas.

realizar un tratamiento temprano y redireccionar el crecimiento; Sar y cols. ${ }^{14}$ mencionan que la finalidad es obtener una mejoría en la relación de los maxilares. Las técnicas para el tratamiento temprano de una clase III esqueletal, aplican la fuerza directa a los dientes superiores a partir de un aparato cementado en el maxilar y utilizan de anclaje aparatos extraorales apoyados al mentón y la frente; se ha demostrado que la aplicación de fuerzas ortopédicas directo a las suturas circunmaxilares promueven la protracción maxilar y estimula la aposición ósea contribuyendo a la corrección de la clase III esqueletal, no obstante, origina múltiples efectos indeseables como la rotación en sentido contrario a las agujas del reloj del maxilar, postero rotación de la mandíbula, retroinclinación de los dientes inferiores y aumento del ángulo mandibular. ${ }^{14}$

Es por esto, que se han buscado alternativas para tratar las clases III esqueletales; en 1985, Kokich propuso colocar mini implantes en monos para evaluar el anclaje absoluto durante la protracción maxilar, pero no fue hasta el 2009 cuando De Clerck ${ }^{12}$ colocó mini placas de titanio en el maxilar y mandíbula para proveer anclaje absoluto y a partir de estas usar elásticos intermaxilares por 24 horas para obtener una tracción del maxilar, disminuir los efectos dentoalveolares indeseados y rotación mandibular en sentido horario originado con la máscara facial, sin embargo, tiene la desventaja que para su colocación y retiro se requiere de un procedimiento quirúrgico lo cual origina inflamación post operatoria. ${ }^{9,12}$ Cornelis y cols., ${ }^{16}$ demuestran que los efectos verticales con las mini placas

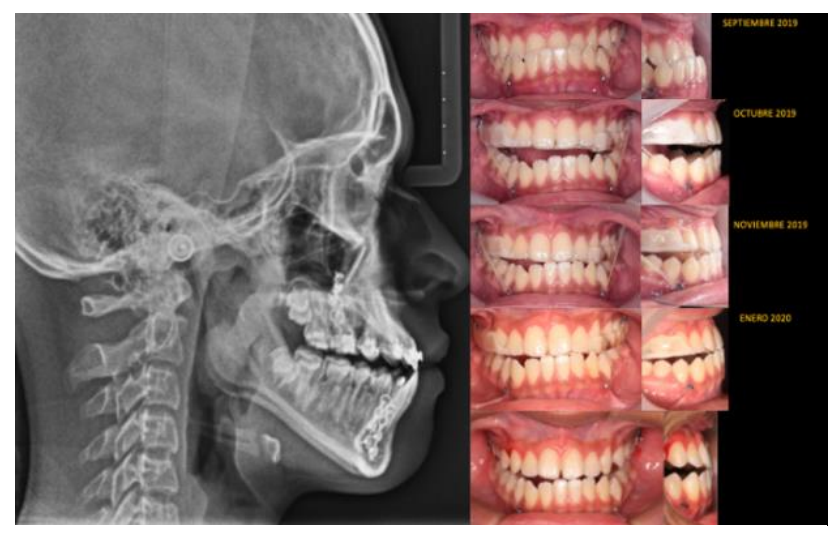

Figura 3. Radiografía final y fotos intraorales comparativas de evolución.

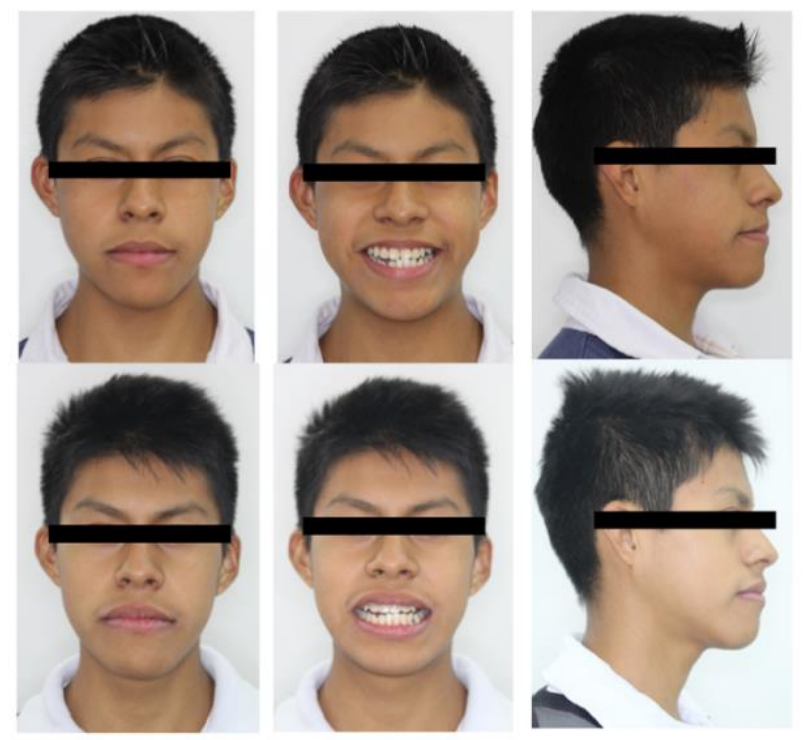

Figura 4. Comparativas extraorales inicial y final. 
son clínicamente insignificantes y Sahin y cols. ${ }^{17}$ reportan nulos efectos dentoalveolares.

Eid y cols., ${ }^{18}$ con la finalidad de corroborar el efecto del protocolo BAMP, evaluó a 20 pacientes entre los 9 y 12 años. Para esto, colocaron cuatro mini placas en los maxilares y dos semanas después fueron activadas con una fuerza inicial de 300 gr por lado con elásticos intermaxilares, la cual se incrementó un mes después a 350 gr, dos meses después a $450 \mathrm{gr}$, con un total de tratamiento de 12 meses y en la comparativa cefalométrica, demostró aumento en la proinclinación de los incivos superiores, resultados similares a los obtenidos en el presente caso clínico. $^{18}$

Por otra parte, J.Van Hevele y cols., ${ }^{19}$ realizó un estudio en 218 pacientes entre 9 y 14 años para evaluar los efectos de las mini placas en pacientes clase III; después de 14 días de la colocación, empezo la tracción con una fuerza de $100 \mathrm{gr}$ por lado y fue aumentando semanalmente hasta lograr una fuerza máxima de $250 \mathrm{gr}$ por lado; donde obtuvieron un éxito del $93.6 \% .{ }^{19}$ Resultados similares a los reportados por Cevidanes y cols.., ${ }^{20}$ en su estudio realizado en pacientes en étapa prepuberal entre los 11 y 12 años; a quienes les colocaron cuatro mini placas modificadas: Dos en el maxilar y dos en la mandíbula; fueron activadas tres semanas después de su colocación con una fuerza inicial de 150 gr por lado, al segundo mes 200 gr y al tercer mes 250 gr; con una duración total de 12 meses. ${ }^{20}$

En algunos pacientes reportan que hubo interferencias oclusales durante el tratamiento, por lo que decidieron colocar un paladar con pistas planas y concluyeron que permitió una mejor tracción. ${ }^{20}$ Por lo cual, en este reporte de caso se decidió colocar un paladar con pistas planas desde un inicio para permitir el correcto deslizamiento. De igual forma, Fakhgarian y cols.., ${ }^{21}$ usaron mini placas modificadas con la finalidad de disminuir costos y lograron una completa corrección esqueletal; en su cefalometría reporta modificaciones en el plano mandibular y palatino. Así mismo, De Clerck y Swennen, ${ }^{22}$ usaron mini placas modificadas en pacientes de 12 años con una fuerza inicial de 150 gr por 12 meses con resultados favorables.

Miranda y cols.., ${ }^{23}$ activaron por lado con elásticos intermaxilares con fuera inicial de150 gr de el mismo día de la colocación y un mes después aumentaron a 250 gr por lado; se les indicó utilizarlos por 24 horas y cambiarlos cada
12 horas; obtuvieron mejoría en la relación esqueletal con mínimos cambios dentales en los incisivos superiores e inferiores. ${ }^{23}$ Por otra parte, Elnagar y cols.., ${ }^{24}$ mencionan que el tratamiento con mini placas es una buena alternativa para pacientes en etapa de dentición mixta-tardía o temprana permanente y recomiendan el uso en conjunto de la máscara facial y si es necesario, expansión maxilar, sin embargo, concluyen que las mini placas brindan disminución del plano mandibular a comparación de la mascara facial.

\section{CONCLUSIONES}

El protocolo de protracción BAMP, es una alternativa para pacientes que están en el por terminar su crecimiento esqueletal y que todavía tengan la posibilidad de mejorar la relación esqueletal maxilo-mandibular; no obstante, no en todos los casos se obtienen los resultados deseados, sin embargo, se aprovecha el crecimiento y logra mejorar la discrepancia dental y esqueletal lo que permite un tratamiento a futuro menos complejo y en el mejor de los casos, evita procedimientos quirúrgicos al llegar a la edad adulta.

A pesar de que este protocolo es indicado en conjunto al uso de la máscara facial, se decidió no utilizarla para disminuir el impacto psicológico que podría causar al paciente. A pesar de esto, los resultados fueron los esperados; se reportó mejoría en el overjet, overbite y debido al impacto que hubo la posición del maxilar, se obtuvo mejoría en la posición del labio superior. Como segunda fase, se recomienda continuar con el tratamiento ortodóncico y si el caso lo requiere, realizar patrón de extracciones clase III de premolares; y continuar el tratamiento de forma simultánea con el uso de elásticos intermaxilares hacia las mini placas. El paladar con pistas planas se reemplazará con topes oclusales en las primeras y segundas molares inferiores para seguir permitiendo el deslizamiento generado por los elásticos y lograr los objetivos propuestos para esta segunda etapa.

\section{AGRADECIMIENTOS}

Los autores queremos agradecer a la Facultad de Estomatología y al Posgrado de Ortodoncia de la Benemérita Universidad Autónoma de Puebla, en especial al Dr. Alejandro Dib Kanan, Dr. Farid A. Dipp Velázquez 
y Dr. Juan Fernando Aristizabal por permitirnos hacer uso de las instalaciones y equipo de la clínica de la BUAP y de la Universidad del Valle. Paloma González Higuera agradece al CONACyT y al Programa de Becas para Estudios de Posgrado.

\section{REFERENCIAS}

1. Espinar-Escalona E, Ruiz-Navarro MB, Ortega-Rivera H, Llamas-Carreras JM, Barrera-Mora JM, Solano-Reina JE. Tratamiento temprano de las Clases III. Rev Esp Ortod. 2011;41:79-89.

2. Ramírez-Mendoza J, Muñoz-Martínez C, Gallegos-Ramírez A, Rueda-Ventura MA. Maloclusión clase III. Salud en Tabasco. 2010;944-50.

3. Reyes-Ramírez DL, Etcheverry-Doger E, Antón-Sarabia J, Muñoz-Quintana G. Asociación de maloclusiones clase I, II y III y su tratamiento en población infantil en la ciudad de Puebla, México. Revista Tamé. 2014;2(6):175-9.

4. Zere E, Chaudhari PK, Sharan J, Dhingra K, Tiwari N. Developing Class III malocclusions: Challenges and solutions. Clin Cosmet Investig Dent. 2018;10:99-116.

5. Clemente R, Contardo L, Greco C, Di Lenarda R, Perinetti G. Class III Treatment with Skeletal and Dental Anchorage: A Review of Comparative Effects. Biomed Res Int. 2018;2018:110.

6. D’Escriván de Saturno L. Ortodoncia en dentición mixta. 2da Edició. Orthodontics WJ of, editor. Venezuela: Amolca; 2007. $1-555 \mathrm{p}$.

7. Ngan P, Wilmes B, Drescher D, Martin C, Weaver B, Gunel E. Comparison of two maxillary protraction protocols: toothborne versus bone-anchored protraction facemask treatment. Prog Orthod. 2015;16(26):1-11.

8. Pereira da Silva HCF, de Paiva JB, Rino Neto J. Anterior crossbite treatment in the primary dentition: Three case reports. Int Orthod. 2018;16(3):514-29.

9. Proffit WR, Fields HW, Sarver DM, Ackerman JL. Ortodoncia Contemporánea. 5ta Edició. Elsevier, editor. España; 2013. 770 p.

10. Martinez Smit R, Aristizábal JF. Maloclusión Clase III : Diagnóstico y Tratamiento Ortopédico . Revisión de Literatura y Reporte de Caso. Revista Cientifica Sociedad de Ortodoncia. 2016;3(2):7-17.

11. De-Clerck H, Cevidanes L, Baccetti T. Dentofacial effects of bone-anchored maxillary protraction: A controlled study of consecutively treated Class III patients. J Orthod Dentofac Orthop. 2010;138(5):577-581.

12. De Clerck H, Cornelis MA, Cevidanes LH, Heymann GC, Tulloch CJF. Orthopedic Traction of the Maxilla with Miniplates: A New Perspective for Treatment of Midface Deficiency. J Oral Maxillofac Surg. 2009;67(10):2123-9.

13. Baccetti T, Franchi L, McNamara JA. The Cervical Vertebral Maturation (CVM) method for the assessment of optimal treatment timing in dentofacial orthopedics. Semin Orthod. 2005;11(3):119-29.
14. Şar Ç, Arman-Özçirpici A, Uçkan S, Canan-Yazici A. Comparative evaluation of maxillary protraction with or without skeletal anchorage. Am J Orthod Dentofac Orthop. 2011;139(5):636-49.

15. Degala S, Bhanumathi M, Shivalinga BM. Orthopaedic Protraction of the Maxilla with Miniplates: Treatment of Midface Deficiency. J Maxillofac Oral Surg. 2015;14(1):111-8.

16. Cornelis MA, Tepedino M, De Vos-Riis N, Niu X, Cattaneo PM. Treatment effect of bone-anchored maxillary protraction in growing patients compared to controls: a systematic review with metaanalysis. Eur J Orthod. 2020 Aug 20;1-18.

17. Sahin T, Delforge A, Garreau E, Raoul G, Ferri J. Orthopedic treatment of Class III malocclusions using skeletal anchorage: A bibliographical review. Int Orthod. 2016;14(3):263-72.

18. Mohamed-Eid O, Abdel-Fattah Ramadan A, Adel-Nadim M, AbdelBary-Hamed T. Maxillary protraction using orthodontic miniplates in correction of Class III malocclusion during growth. J World Fed Orthod. 2016;5(3):100-6

19. Van Hevele J, Nout E, Claeys T, Meyns J, Scheerlinck J, Politis C. Bone-anchored maxillary protraction to correct a class III skeletal relationship: A multicenter retrospective analysis of 218 patients. J Cranio-Maxillofacial Surg. 2018;46(10):1800-6.

20. Cevidanes L, Baccetti T, Franchi L, McNamara JA, De Clerck H. Comparison of two protocols for maxillary protraction: Bone anchors versus face mask with rapid maxillary expansion. Angle Orthod. 2010;80(5):799-806.

21. Fakharian M, Bardideh E, Abtahi M. Skeletal class iii malocclusion treatment using mandibular and maxillary skeletal anchorage and intermaxillary elastics: A case report. Dental Press J Orthod. 2019;24(5):52-9.

22. De Clerck H, E.B E, Swennen G-RJ. Success rate of miniplate anchorage for bone anchored maxillary protraction. Angle Orthod. 2011;81(6):1010-3.

23. 23. Miranda F, Da Cunha-Bastos JC, Magno-Dos Santos A, Vieira LS, Aliaga-Del Castillo A, Janson G, y cols.. Miniscrew-anchored maxillary protraction in growing Class III patients. J Orthod. 2020;47(2):170-80

24. 24. Elnagar MH, Elshourbagy E, Ghobashy S, Khedr M, Evans CA. Comparative evaluation of 2 skeletally anchored maxillary protraction protocols. Am J Orthod Dentofac Orthop [Internet]. 2016;150(5):751-62. Available from: http://dx.doi.org/10.1016/j.ajodo.2016.04.025 